\author{
Department of Mathematics \\ University of North Texas \\ Denton, TX 76203 \\ January 28, 2013
}

\title{
SOME PROBLEMS AND IDEAS OF ERDÖS IN ANALYSIS AND GEOMETRY
}

\author{
R. DANIEL MAULDIN
}

\begin{abstract}
I review a meager few of the many problems and ideas Erdős proposed over the years involving a mixture of measure theory, geometry, and set theory.
\end{abstract}

\section{INTRODUCTION}

I have selected a few topics from Erdös' many problems and ideas in this area. Some were selected just for the sake of promoting them and others because they have led to several developments and connections. Three sources for some additional problems of Erdôs in these areas may be found in $[17,4,14]$.

\section{Similar COPIES OF SEQUenCes.}

Even in his article of 1978 in [17], Erdös says he had made the following conjecture for a long time:

Conjecture 2.1. Let $\left\{x_{n}\right\}$ be a sequence of positive numbers decreasing to 0 . Is there a Lebesgue measurable set $E$ with positive measure which does not contain any affine copy of the sequence?

In his lecture at the Scottish Book conference in 1979, Erdös said that the problem has been open for so long that he should offer $\$ 100$ for its solution. He also said at that time that he didn't think the problem was difficult. However, this well known and much studied problem remains open. This problem is discussed in some detail in [21] and more recently in the survey article [32].

\section{AdDitive NUMBER THEORY AND EFFECTIVE DIMENSION.}

Erdős conjectured that to each infinite set of positive integers $A$, there corresponds a complementary set $B$, an infinite set of positive integers $B$ with density 0 such that the sum set $A+B$ contains every sufficiently large integer. Lorentz proved the conjecture in [1]. In fact, 
letting $A(n)$ be the number of elements of $A$ not exceeding $n$, Lorentz proved the following

Theorem 3.1. There is a constant c such that every infinite set $A \subset \mathbb{N}$, there corresponds an infinite set $B \subset \mathbb{N}$ such that $A+B$ contains every sufficiently large integer and for each $n$ :

$$
B(n) \leq c \sum_{k=1}^{n} \frac{\log A(k)}{A(k)} .
$$

Inequality (1) clearly shows $B$ has density 0 . Erdös in [3] shows that inequality (1) is the best possible if one only takes into account the rate of increase of $A(n)$ but not its structural properties:

Theorem 3.2. There is a sequence $A$ of positive integers with positive lower density such that for every complementary set $B$ satisfies $B(n)>$ $C_{1}(\log n)^{2}$. This is in agreement with estimate (1).

Erdős also made an improvement if $A$ is the set of primes. For this set, Lorentz's estimate yields the existence of a complementary set $B$ with $B(n)<C_{2}(\log n)^{3}$. Erdös shows there is some $B$ with $B(n)<$ $C_{3}(\log n)^{2}$.

By the way, in [3] Erdös posed the following problem.

Problem 3.3. Is there a set $B$ of positive integers with $B(n)<C_{4} \frac{n}{\log n}$ such that the sets $B+2^{k}$ cover all but finitely many positive integers?

In [33] Ruzsa gave an affirmative answer and later in [34], he even determined the best constant

Lorentz proceeds to prove Theorem 3.1 by first proving a finite version of it:

Theorem 3.4. There is a constant $C$ such that if $m$ and $n$ are integers, $k$ is a positive integer, and $A$ is a set of integers with $A \subset[m, m+k)$ with $\operatorname{card}(A) \geq l \geq 2$, then there are integers $b_{1}<b_{2}<\ldots<b_{K}$ in the interval $(n-k, n+k)$ such that the translates $A+b_{i}$ cover the integers in the interval $(m+n, m+n+k)$ and

$$
K \leq C k \frac{\log l}{l} .
$$

The idea is to select the $b^{\prime} s$ greedily and estimate the number of steps required until the interval is covered. An immediate consequence of Lorentz's estimate is: 
Theorem 3.5. Let $n$ be a positive integer. If $a_{1}, \ldots, a_{l}$ is a set of incongruent residues modulo $n$, there is another set of residues $b_{1}, \ldots, b_{k}$ with

$$
k \leq C n \frac{\log l}{l}
$$

such that each residue modulo $n$ is of the form $a_{i}+b_{j}$.

If one would like to somehow measure the structural properties of $A$, a finite set of, say, positive integers with cardinality at least 2 , one could consider what Randall Dougherty calls $\operatorname{den}_{\text {cover }}(A)$, the 'covering density of $A$.' This is defined as follows. For each $n$, let $C(A, n)$ be the minimal number of translated copies of $A$ needed to cover $[1, n] \cap \mathbb{N}$. Then

$$
\operatorname{den}_{\text {cover }}(A)=\lim _{n \rightarrow \infty} \frac{\operatorname{card}(A) C(A, n)}{n} .
$$

Clearly, den $n_{\text {cover }}(\{1,2,3\})=1$. But, den $n_{\text {cover }}(\{1,2,4\})=6 / 5$.

In another direction, Erdős, Kunen and I in [13] used Lorentz's theorem to prove the following:

Theorem 3.6. Let $P$ be a nonempty perfect subset of $\mathbb{R}$. Then there is a perfect set $M$ with Lebesgue measure zero such that $P+M=\mathbb{R}$.

One could consider extensions of these theorems and ideas to groups other than $\mathbb{R}$.

P. Elias in [9] has obtained a stronger form of Theorem 3.6. Using Kronecker's approximation theorem, he has shown that the set $M$ of the theorem may be taken to be Dirichlet set. A set $M$ is said to be a Dirichlet set if there exists an increasing sequence of positive integers $\left\{n_{k}\right\}$ such that the sequence of functions $\left\{\sin n_{k} x\right\}$ converges uniformly to 0 on $M$.

Also, Lorentz's theorem has a direct application in effective geometric measure theory. The Kolmogorov complexity of a string $\sigma$, denoted $K(\sigma)$, is the length (in this paper we will measure length in ternary units) of the shortest program (under a fixed universal machine) which outputs $\sigma$ [16]. For a real number $x, x \uparrow n$ denotes the first $n$ digits in a ternary expansion of $x$. Martin-Löf random reals have high initial segment complexity [23]; indeed every Martin-Löf random real $r$ satisfies $\lim _{n} K(r \uparrow n) / n=1$. This fact conforms with our intuition that the M-L random objects do not compress much.

Recall some classical dimension notions. Let $E \subseteq \mathbb{R}^{n}$. The diameter of $E$, denoted $|E|$, is the supremum of the distances between any two points in $E$. A cover $\mathcal{G}$ for a set $E$ is a collection of sets whose union contains $E$, and $\mathcal{G}$ is a $\delta$-mesh cover if the diameter of each member 
$\mathcal{G}$ is at most $\delta$. For a number $\beta \geq 0$, the $\beta$-dimensional Hausdorff measure of $E$, written $\mathcal{H}^{\beta}(E)$, is given by $\lim _{\delta \rightarrow 0} \mathcal{H}_{\delta}^{\beta}(E)$ where

(5) $\mathcal{H}_{\delta}^{\beta}(E)=\inf \left\{\sum_{G \in \mathcal{G}}|G|^{\beta}: \mathcal{G}\right.$ is a countable $\delta$-mesh cover of $\left.E\right\}$.

The Hausdorff dimension of a set $E$, denoted $\operatorname{dim}_{\mathrm{H}}(E)$, is the unique number $\alpha$ where the $\alpha$-dimensional Hausdorff measure of $E$ transitions from being negligible to being infinitely large; if $\beta<\alpha$, then $\mathcal{H}^{\beta}(E)=$ $\infty$ and if $\beta>\alpha$, then $\mathcal{H}^{\beta}(E)=0$ [26].

The effective (or constructive) $\beta$-dimensional Hausdorff measure of a set $E, c \mathcal{H}^{\beta}(E)$, is defined exactly in the same way as Hausdorff measure with the restriction that the covers be uniformly c.e. (= computably enumerable) open sets [23, Definition 13.3.3]. This yields the corresponding notion of the effective (or constructive) Hausdorff dimension of a set $E, \operatorname{cdim}_{\mathrm{H}} E$. Lutz [22] showed that constructive dimension of a set is determined by the constructive dimension of its points:

$$
\operatorname{cdim}_{\mathrm{H}} E=\sup \left\{\operatorname{cdim}_{\mathrm{H}}\{x\}: x \in E\right\},
$$

and from work of Mayordomo $[19](\geq)$ and Levin $[20](\leq)$ (also see [23]) we have for any real number $x$,

$$
\operatorname{cdim}_{\mathrm{H}}\{x\}=\liminf _{n \rightarrow \infty} \frac{K(x\lceil n)}{n} .
$$

We define the constructive dimension of a point $x$ to be the effective Hausdorff dimension of the singleton $\{x\}$. In [18], Lorentz's theorem plays a central role in the proof of the following.

Theorem 3.7. Let $C$ be the standard middle-third Cantor set. For any $\alpha$ satisfying $1-\operatorname{dim}_{\mathrm{H}}(C) \leq \alpha \leq 1$, and for any Martin-Löf random $r \in[0,1]$, we have

$$
\operatorname{dim}_{H}\left((C+r) \cap E_{=\alpha}\right)=\operatorname{dim}_{H}\left((C+r) \cap E_{\leq \alpha}\right)=\alpha-1+\operatorname{dim}_{\mathrm{H}}(C),
$$

where $E_{=\alpha}$ consists of all real numbers with constructible dimension $\alpha$ and $E_{\leq \alpha}$ is the set of reals of dimension at most $\alpha$.

The constructive dimension of $\left.(C+r) \cap E_{=\alpha}\right)$ is $\alpha$ whereas the Hausdorff dimension of this set is $\alpha-1+\operatorname{dim}_{\mathrm{H}}(C)$. This means that for a given M-L random real $r$ there are many points $x$ in the Cantor set which cancels the randomness of $r$, i.e., $x+r$ has lower constructive dimension; the initial strings of $r+x$ have a factor less Kolmogorov complexity than the corresponding initial strings of $r$. 
It seems that we have just begun to delve into the possibilities in this direction. For example, one could investigate analogues of Theorem 3.7 for other totally disconnected self similar or self conformal sets in $\mathbb{R}$ or $\mathbb{R}^{n}$.

\section{Dimension of Subgroups And Rings}

Erdős and Volkmann in [12] proved the following theorem.

Theorem 4.1. For each $\alpha$ with $0<\alpha<1$, there is an additive Borel subgroup of the reals with Hausdorff dimension $\alpha$.

Several proofs of this fact have now been given. They all involve some set of numbers which are well approximated by rationals. For example, (see [26]), fix $0<\alpha<1$ and let $n_{k}$ be a sequence of positive integers which increases sufficiently rapidly. Let

$$
x \in G \Longleftrightarrow \exists M \forall k \exists \text { integer } p:\left|x-\frac{p}{n_{k}}\right|<\frac{M}{n_{k}^{\alpha}} .
$$

Clearly, $G$ is an additive subgroup of $\mathbb{R}$ and it can be shown that $\operatorname{dim}_{\mathrm{H}}(G)=\alpha$. However, if one asks about subrings of $\mathbb{R}$, Edgar and Miller [25] showed the answer is quite different.

Theorem 4.2. If the Borel set $F$ is a subring of $\mathbb{R}$, then either $\operatorname{dim}_{H}(F)=$ 0 or $\operatorname{dim}_{H} F=1$.

In fact, Edgar and Miller show that

Theorem 4.3. If the Borel set $F$ is a subring of $\mathbb{C}$, then either $\operatorname{dim}_{H}(F)=$ 0 or $F=\mathbb{R}$ or $F=\mathbb{C}$.

This leads to the following problem.

Problem 4.4. For which $\alpha$ other than 0,1 or 2 are there subrings of $\mathbb{R}$ or $\mathbb{C}$ with Hausdorff dimension $\alpha$ ?

This is really a question about transfinite constructions. Things are not so clear for other rings. Consider the example of D. Goldstein.

Example 4.5. Let the Borel set $G$ be an additive subgroup of $\mathbb{R}$ with $\operatorname{dim}_{H}(G)=\alpha$. Let $F$ consist of all $2 \times 2$ matrices $M$ of the form

$$
M=\left[\begin{array}{cc}
m & x \\
0 & n
\end{array}\right]
$$

where $x \in G$ and $m, n \in \mathbb{Z}$.

Then for any matrix norm, we have for the Borel subring $F, \operatorname{dim}_{\mathrm{H}}(F)=$ $\alpha$. 
Thus, for every $\alpha$ with $0 \leq \alpha \leq 1$, there are Borel subrings of the space of $2 \times 2$ with dimension $\alpha$. But we don't know the answer for larger $\alpha$.

Problem 4.6. For which $\alpha>1$ does the space of $2 \times 2$ real valued matrices have a (Borel) subring with Hausdorff dimension $\alpha$ ? Of course, one can consider this problem in a more general context.

Buhler, Butler, de Launey and Graham in [24] investigated 'Origami rings' in $\mathbb{C}$ generated as follows. Let $L_{\alpha}(p)$ be the line in the complex plane through $p$ with angle $\alpha$. Given a collection $U$ of angles, let $R(U)$ be the points that can be obtained by starting with 0 and 1 , and then recursively adding intersection points of the form $L_{\alpha}(p) \cap L_{\beta}(q)$, where $p, q$ have been already been generated, and $\alpha, \beta$ are in $U$ and the lines are distinct. For each $n$, let $U_{n}$ be the group of the $n$ equally spaces angles $k \pi / n, 0 \leq k<n$. They characterize the subrings of $\mathbb{C}$ generated by the finite subgroups $U$ where $3 \leq \operatorname{card}(U)$ as follows.

Theorem 4.7. Let $n \geq 3$ If $n$ is prime, the $R\left(U_{n}\right)=\mathbb{Z}\left[\zeta_{n}\right]$, the cyclotomic integer ring. If $n$ is not a prime, then $R\left(U_{n}\right)=\mathbb{Z}\left[1 / n, \zeta_{n}\right]$, the cyclotomic integer ring localized aat the primes dividing $n$. Moreover, if $N>3$, then $R\left(U_{n}\right)$ is dense in the plane.

This led Goldstein and I to construct uncountable subgroups $G$ of the circle group which are the union of countably many compact sets each with box counting dimension 0 (Actually, such subgroups had been constructed much earlier by Laczkovich and Ruzsa in [28].) It follows from this that the subring of $\mathbb{C}$ generated by $G$ still has Hausdorff dimension 0 . This leads to the following problem.

Problem 4.8. Is there a subgroup $G$ of the circle group with $\operatorname{dim}_{\mathrm{H}}(G)=$ 0 such that $G$ is not the union of countably many sets with lower box counting dimension 0 and yet the ring generated by $G$ still has dimension 0 ?

\section{Sets containing the vertices of a triangle of AREa 1}

Many years ago Erdős noted that if $E$ is a Lebesgue measurable subset of the plane with infinite measure, then for every $c>0, E$ contains the vertices of a triangle of area $c$. As several people have noted, this remains true if $E$ has positive measure and is unbounded.

In [17] and again in $[4,14]$, Erdős poses what he said was an interesting and perhaps difficult problem, even though, as far as I know, he never did offer any money for its solution. 
Problem 5.1. Is there a finite constant $C$ such that if a Lebesgue measurable set $E$ has measure greater than $C$, then $E$ contains the vertices of a triangle of area 1? Moreover, is it true that the best constant is $c_{0}=4 \pi / 3 \sqrt{3}$, the area of the disk such that the area of the inscribed equilateral triangle is 1 ?

Chris Freiling and I have studied this problem. Using some standard approximations in measure theory, Erdős' problem is equivalent to the following problem.

Problem 5.2. Is there a finite constant $c$ such that for every $n \in \mathbb{N}$ if $E$ is the union of the interiors of no more than $n$ compact convex sets and $E$ has measure greater than $c$, then $E$ contains the vertices of a triangle of area 1 . Moveover, is $c_{0}$ the best possible constant?

We showed in [29] that the constant $c_{0}$ is the best possible if $n$ is 1 . I reiterate the argument here. Suppose one has then a compact convex set $K$ of positive area which is "small" meaning $K$ does contain the vertices of a triangle of area greater than 1 . If one takes a line $l$, then the Steiner symmetrical of $K$ about $l$ has the same area as $K$ and also does not contain the vertices of a triangle of area greater than 1 . There is a sequence $K_{n}$, each of which is obtained by iterating the process of taking Steiner symmetrizations of $K$ about a finite number of lines through the origin which converges to the closed disk centered at the origin with the same area as $K$, (see [31]). From this, it follows that the area of $K$ is no more than $c_{0}$. So, Erdös' conjecture is true if $n=1$.

Let $E$ be the union of the interiors of the compact convex sets $K_{1}, \ldots, K_{n}$ and suppose $E$ does not contain the vertices of a triangle of area 1 . Then the area of any triangle whose vertices belong to two of the sets $K_{i}$ must be less than 1 . If $i, j, k$ are different, then either the area of every triangle with one vertex from each of $K_{i}, K_{j}, K_{k}$ is at most 1 , or the areas of all such triangles is at least 1.

For $n=2, c_{0}$ is still the best constant. If we have two compact convex bodies $K_{1}$ and $K_{2}$ such that their union does not contain the vertices of a triangle of area greater than 1 , then their compact convex hull doesn't either, (see [29]).

Even for $n=3$, we may argue $c_{0}$ is the best constant. Suppose $E=E_{1} \cup E_{2} \cup E_{3}$, where each set $E_{i}$ is the interior of a compact convex set $K_{i}$. If $E_{1}, E_{2}$, and $E_{3}$ form a "small" triple, i.e., the area of every triangle with its vertices in different sets $E_{i}$ has area less than 1 , then since their closed convex hull would have no triangle with area greater than 1 (see [29]), we are reduced to the case $n=1$. On the other hand, if $E_{1}, E_{2}$, and $E_{3}$ form a "large" triple, we use the following 
redistribution of mass argument. Let us suppose $K_{1}$ has the smallest area of the three bodies. There must be a line $L$ which supports both $K_{2}$ and $K_{3}$ such that $E_{2}$ and lie in one half plane determined by $L$ and $K_{1}$ lies in the interior of the other half plane. Let $A \in L \cap K_{2}$ and $B \in L \cap K_{3}$. Let $C \in K_{1}$. The triangle with vertices $A, B$ and $C$ must have area at least 1 . Let us take lines $l$ parallel to $L$ and cutting the interior of both $K_{2}$ and $K_{3}$. The line $l$ intersect $K_{2}$ in points $A_{1}$ and $A_{2}$ and meets $K_{3}$ in points $B_{1}$ and $B_{2}$, where $A_{2}$ and $B_{1}$ are closer together than $A_{1}$ and $B_{2}$. Since the area of triangle $A_{1} A_{2} C$ is no more than one and the area of triangle $A_{2} B_{1} C$ is at least 1 , $\left\|A_{1}-A_{2}\right\| \leq\left\|A_{2}-B_{1}\right\|$. Similarly, $\left\|B_{1}-B_{2}\right\| \leq\left\|A_{2}-B_{1}\right\|$. This is so for lines $l$ until we reach a line that is a support line to either $K_{2}$ or to $K_{3}$. In either case, this implies the area we have swept out between $K_{2}$ and $K_{3}$ is at least the area of the smaller of the areas of $K_{2}$ and $K_{3}$ and therefore the area is at least as large as the area of $K_{1}$. So, if we replace the three bodies $K_{1}, K_{2}$, and $K_{3}$ with the single body formed by $K_{2}, K_{3}$ and the area between them, we are back to the case $n=1$.

\section{Partitions of Lines and PLanes}

In generalizing a result of Sierpinski, Erdős in [11] proved the following.

Theorem 6.1. The following two statements are equivalent:

(1) $\mathrm{CH}$, the continuum hypothesis holds: $2^{\omega}=\omega_{1}$.

(2) If the lines in $\mathbb{R}^{2}\left(\mathbb{R}^{3}\right)$ are colored with 2 colors, then there exists a coloring of $\mathbb{R}^{2}\left(\mathbb{R}^{3}\right)$ with the same colors such that each line contains only countably many points with its color.

Erdős, Jackson and I answered one of Erdős' question in [11] by proving the following.

Theorem 6.2. The following two statements are equivalent:

(1) $\mathrm{CH}$, the continuum hypothesis holds: $2^{\omega}=\omega_{1}$.

(2) If the lines in $\mathbb{R}^{2}\left(\mathbb{R}^{3}\right)$ are colored with three colors, then there exists a coloring of $\mathbb{R}^{2}\left(\mathbb{R}^{3}\right)$ with the same colors such that each line contains only finitely many points with its color.

These results and several others involving flats in $\mathbb{R}^{n}, n \geq 2$ are discussed in [29]. Recently, Humke and Laczkovich used Erdős's original result to show that assuming $\mathrm{CH}$ holds there are subsets of the plane with some very unusual linear density properties [27]. One can imagine that there are several other types of strange examples using other partition results. 


\section{ExaCt Dimension of CONTINUED FRACTIONS USING ONLY THE PRIMES}

In [30], Urbanski and I studied $S_{I}$, the set of continued fractions of the form

$$
\frac{1}{b_{1}+\frac{1}{b_{2}+\frac{1}{b_{3}+\frac{1}{\ddots}}}}
$$

where $I$ is a fixed subset of $\mathbb{N}$ and each $b_{n} \in I$. We developed a pressure function which allowed us to determine the Hausdorff dimension $\alpha=\alpha_{I}$ of $S_{I}$. We showed that for those sets $I$ for which the pressure function has a zero, there is a natural conformal probability measure supported on $S_{I}$ and a corresponding Gauss measure, a measure supported on $S_{I}$ equivalent to the conformal measure and which is invariant under the shift map on $S_{I}$. Using futher properties such as the generalized density of $I$ we found some conditions to determine whether $\mathcal{H}^{\alpha}\left(S_{I}\right)$ is 0 , positive and finite, or $\infty$. We also found some conditions such as some properties of the gaps in $I$ which help to determine whether the $\alpha$-dimensional packing measure $\mathcal{P}^{\alpha}\left(S_{I}\right)$ is 0 , positive and finite, or $\infty$. For example, if $p \geq 2$ and $I=\left\{n^{p}: n \in \mathbb{N}\right\}$, then $0<\mathcal{H}^{\alpha}\left(S_{I}\right)<\infty$ and $\mathcal{P}^{\alpha}\left(S_{I}\right)=\infty$. On the other hand, if $I$ has bounded gaps, then $\mathcal{P}^{\alpha}\left(S_{I}\right)<\infty$. If $I$ is the set of primes, using Erdős' theorem that there are arbitrarily large two sided gaps in the sequence of primes [2], we showed that there is a conformal measure and a corresponding Gauss measure for this system, and yet $0=\mathcal{H}^{\alpha}\left(S_{I}\right)$ and $\mathcal{P}^{\alpha}\left(S_{I}\right)=\infty$. A natural question which we posed in [30] is:

Problem 7.1. Let $S$ be the set of all standard continued fractions of the form

$$
\frac{1}{b_{1}+\frac{1}{b_{2}+\frac{1}{b_{3}+\frac{1}{\ddots}}}}
$$

where each $b_{i}$ is a prime. Is there a Hausdorff gauge function $g$ of the form $g(t)=t^{\alpha} L(t)$, where $L(t)$ is slowly varying such that $0<\mathcal{H}^{g}(S)<$ $\infty$ ? 
If $I$ is a finite subset of $\mathbb{N}$ or if $I=\mathbb{N}$, then both $\mathcal{H}^{\alpha}\left(S_{I}\right)$ and $\mathcal{P}^{\alpha}\left(S_{I}\right)$ are positive and finite. We also don't know the answer to the following problem:

Problem 7.2. Is there a proper infinite subset I of $\mathbb{N}$ such that both $\mathcal{H}^{\alpha}\left(S_{I}\right)$ and $\mathcal{P}^{\alpha}\left(S_{I}\right)$ are positive and finite?

\section{REFERENCES}

[1] G. G. Lorentz, On a problem of additive number theory, Proc. Amer. Math. Soc. 5 (1954), 838-841.

[2] P. Erdős, On the difference of consecutive primes, Quart. J. Oxford 6 (1935), 124-128.

[3] P. Erdős, Some results on additive number theory, Proc. Amer. Math. Soc. $5(1954), 847-853$.

[4] P. Erdős, My Scottish Book Problems, in: The Scottish Book, Mathematics from the Scottish Café. Edited by R. Daniel mauldin, Birkhäuser, Boston, Mass., 1981.

[5] D. Khoshnevisan, Probability, Graduate Studies in Mathematics, AMS, 2007.

[6] Z. Buczolich and R. D. Mauldin, On the convergence of series of translates for measurable functions, mathematika, 46 (1999), 337-341.

[7] Z. Buczolich, J.-P. Kahane, and R. D. Mauldin, On series of translates of positive functions, Acta Math. Hungarica 98 (2001), 171-188.

[8] Z. Buczolich and R. D. Mauldin, On series of translates of positive functions II, Indagationes Math. 12 (2001), 317-327.

[9] P. Elias, Dirichlet sets, Erdős-Kunen-Mauldin theorem and analytic subgroups of the reals. Proc. Amer. Math. Soc. 139, (2010), 2093-2104.

[10] P. Erdős, On the strong law of large numbers, Trans. Amer. Math. Soc., 67 (1949), 51-56.

[11] P. Erdős, Some remarks on set theory. IV, Mich Math. J. 2 (1953-54), 169-173 (1955).

[12] P. Erdős and B. Volkmann, Additive Gruppen mit vorgegebener Hausdorffscher dimension. J. Reine Angew. Math. 221 (1966), 203-208.

[13] P. Erdős, K. Kunen, R. D. Mauldin, Some additive properties of sets of real numbers, Fund. Math. 113 (1981), 187-199.

[14] P. Erdős, Some combinatorial, geometric and set theoretic problems in measure theory, in Measure Theory, Oberwolfach 1983, Lecture Notes in Mathematics 1089, Springer-Verlag (1984).

[15] K. J. Falconer, The geometry of fractal sets, Cambridge Tracts in Mathematics, vol. 85, Cambridge university press, Cambridge, 1986.

[16] Ming Li and Paul Vitanyi, An introduction to Kolmogorov complexity and its applications, third ed., Texts in Computer Science, Springer, New York, 2008.

[17] P. Erdős, Set-theoretic, measure-theoretic, combinatorial, and numbertheoretic problems concerning point sets in Euclidean space, Real Anal. Exchange 4, no. 2, (1978/79), 113-138.

[18] R. Dougherty, J.H. Lutz, R. D. Mauldin, J. Teutsch, Translating the Cantor set by a random real, Trans. Amer. Math. Soc., to appear.

[19] Elvira Mayordomo, A Kolmogorov complexity characterization of constructive Hausdorff dimension, Inform. Process. lett. 84 (2002), no. 1, 1-3. 
[20] L. A. Levin, The concept of a random sequence, Dokl. Akad. Nauk SSSR 212 (1973), 548-550.

[21] H.T. Croft, K.J. Falconer, Richard K. Guy, Unsolved problems in geometry, Springer-Verlag, Berlin, 1994.

[22] J. H. Lutz, The dimensions of individual strings and sequences, Inform. and Comput. 187 (2003), no. 1, 49-79.

[23] R. G. Downey and D. R. Hirschfeldt, Algorithmic randomness and complexity, Theory and Applications of Computability, Springer, New York, 2010.

[24] J. P. Buhler, S. Butler, W. de Launey, R. Graham, Rings Arising in Oragami, J. Australian Math. Soc., to appear.

[25] G. A. Edgar and C. Miller, Borel subrings of the reals, Proc. Amer. Math. Soc., 131, 1121-1129, 2002.

[26] K. Falconer, Fractal Geometry, John Wiley \& Sons Inc., Hoboken, NJ, second edition, 2003.

[27] P. D. Humke, M. Laczkovich, Transference of Density, preprint, 2012.

[28] M. Laczkovich and I. Z. Rusza, Measure of sumsets and ejective sets I., Real Analysis Exchange 22 (1996-1997), 153-166.

[29] R. D. Mauldin, Some problems in set theory, analysis and geometry, in Paul Erdős and his Mathematics I, 493-505, Springer, 2002.

[30] R. D. Mauldin and M. Urbanski, Conformal iterated function systems with applications to the geometry of continued fractions, Trans. Amer. Math. Soc. 351 (1999), 4995-5025.

[31] Roger Webster, Convexity, Oxford University Press, 1994.

[32] R. E. Svetic, The Erdős similarity problem, Real Analysis Exchange, $26(2)(2000), 525-540$.

[33] I. Z. Ruzsa, On a problem of P. Erdős, Canad. math. Bull. 15 (1972), 309-310.

[34] I. Z. Ruzsa, Additive completion of lacunary sequences, Combinatorics 21 (2001), 279-291. 